\title{
Frame-Based Stereotactic-Guided Excision of Colloid Cyst of Cavum Septum Pellucidum through Transcallosal Approach-A Technical Note
}

\author{
J. K. B. C. Parthiban ${ }^{1} \quad$ S. Shanthanam ${ }^{1} \quad$ R. Manivasagam ${ }^{2}$ \\ ${ }^{1}$ Department of Neurosurgery, Kovai Medical Centre and Hospital, \\ Coimbatore, Tamil Nadu, India \\ ${ }^{2}$ Balaji Neuropsychiatric Clinic, R.S. Puram, Coimbatore, Tamil Nadu, \\ India \\ Indian J Neurosurg 2018;7:235-238
}

\author{
Address for correspondence J. K. B. C. Parthiban, MCh, Department \\ of Neurosurgery, Kovai Medical Center and Hospital, P.B. No. 3209, \\ Avanashi Road, Coimbatore 641014, India \\ (e-mail: juttyparthiban@gmail.com).
}
Abstract
Keywords
- colloid cyst
- cavum septum pellucidum
- stereotactic-guided excision
- transcallosal approach

Colloid cyst is a rare benign intracranial lesion. Due to its embryologic origin and development, it is commonly located within the third ventricle, though it also presents at various locations. Its location within cavum septum pellucidum is rarely reported. Operative approaches to such lesion can be challenging and misleading due to intraoperative variations. However, surgical resection in such locations are simplified and perfected with stereotactic guidance. A case of colloid cyst within the cavum septum pellucidum and the importance of stereotactic guidance in precisely reaching the lesion, thus minimizing the dissection of corpus callosum, are discussed.

\section{Introduction}

Since its first description in 1858 by Wallmann, ${ }^{1}$ controversies have revolved around the management of colloid cyst, especially in indication and approaches. Colloid cysts are benign lesions ${ }^{2}$ constituting approximately 0.5 to $1 \%$ of intracranial lesions. Embryologically, they are mesodermal in origin as an invagination from stomodeum into neuroectoderm. This complex embryologic origin is partly responsible for its variations in its location and behavior and occurrence of associated anomalies. Location of colloid cyst within cavum septum pellucidum is scarcely reported, and use of stereotaxy for precise surgical excision of the lesion in this location is hardly seen in the literature.

\section{Case History and Technique}

A 16-year-old girl, with no prior comorbid illness, was referred with history of headache of 3-month duration, which was initially episodic, random, with no diurnal variation, with no definite periodicity. The headache was holocranial and was not radiating, and there were no precipitating factors. It was also not associated with vomiting or blurring of vision. During this period, the patient had five episodes of transient tonic posture with stare look associated with loss of orientation to place and amnesia subsequently, for which she was on oxcarbazepine. On examination, she was clinically normal, with no focal neurologic deficits. Fundus was essentially normal with no papilledema. She was evaluated elsewhere with a plain and contrast computed tomography (CT) of the brain ( - Fig. 1), which showed a noncontrast-enhancing hyperdense lesion lying within the cavum septum pellucidum, suggesting a colloid cyst. Magnetic resonance imaging (MRI) of the brain (-Fig. 2) was done, which supported the same diagnosis. She was planned for stereotactic-guided resection of the lesion, which was a routine technique done in the unit for all deep-seated lesions. Using CRW (Cosman-Roberts-Wells) frame-based stereotactic guidance, right middle one-third parasagittal craniotomy was done. Interhemispheric fissure was dissected, and both anterior cerebral arteries were identified and played aside. The corpus callosum was dissected at the midline along the trajectory of received

September 11, 2016

accepted

June 29, 2017

published online

October 17, 2017
Dol https://doi.org/

$10.1055 / \mathrm{s}-0037-1606825$

ISSN 2277-954X.
๑)2018 Neurological Surgeons'

Society of India
License terms

(ㅇ)(1) $\Theta \circledast$ 


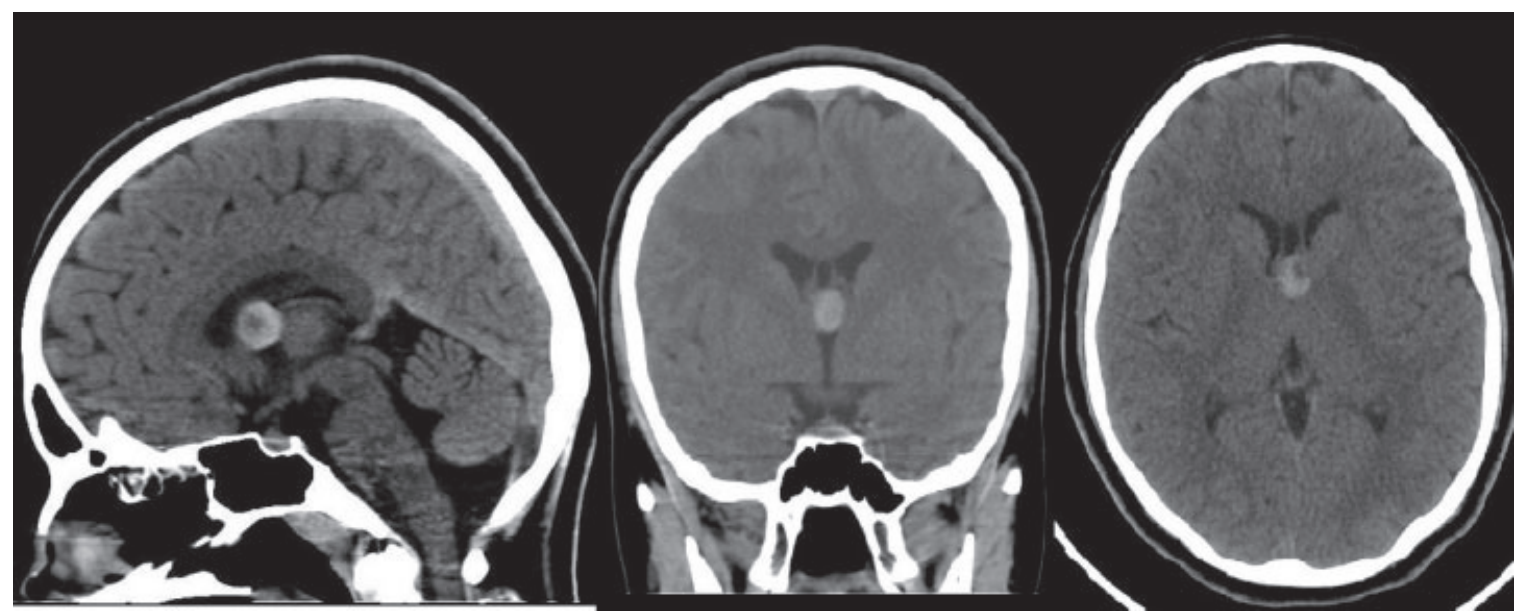

Fig. 1 Preoperative CT sagittal, coronal, and axial sections showing colloid cyst of the cavum septum pellucidum. CT, computed tomography.

the stereotactic probe. Upon dissecting the corpus callosum under stereotactic guidance, the cavum septum pellucidum was entered directly without transgressing the lateral ventricles. The lesion was lying exactly within the cavum with a thin layer of left medial fornix splayed over the lesion that was dissected aside and was excised in total using microsurgical technique. The lesion was capsulated containing yellowish gelatinous fluid. The capsule was attached to the upper surface of the roof of the third ventricle, that is, velum interpositum. Following excision of the lesion, the third ventricular cavity was visible and internal cerebral veins were visible. The stereotactic probe guidance (-Figs. $\mathbf{3}, \mathbf{4}$ ) helped us minimize the length of corpus callosal dissection, enter the cavum septum pellucidum between the two leaflets, and reach the colloid cyst on target. Postoperatively, the patient had three episodes of complex partial seizures, which was controlled with antiepileptics. She improved, and after sixth postoperative day, she was seizure free and discharged. The histopathologic report confirmed the colloid cyst. On review, the patient underwent follow-up CT scan, which showed no residual lesion or hydrocephalus (-Fig. 5).

\section{Discussion}

Colloid cysts are rare benign intracranial lesions, mostly localized in the roof of the third ventricle. Although the cysts are benign histologically, they can present with significant neurologic manifestation, including death due to acute obstructive hydrocephalus.

Colloid cyst wall is built of a collagenous connective tissue stroma, lined with a single layer of stratified epithelium. The cells may be columnar, ciliated and nonciliated, cuboidal, or squamous. The cyst consists of gelatinous material. $^{3}$

The embryologic origin of colloid cyst is controversial, and many theories revolve around its pathogenesis, as also its clinical presentation and operative indications. Small

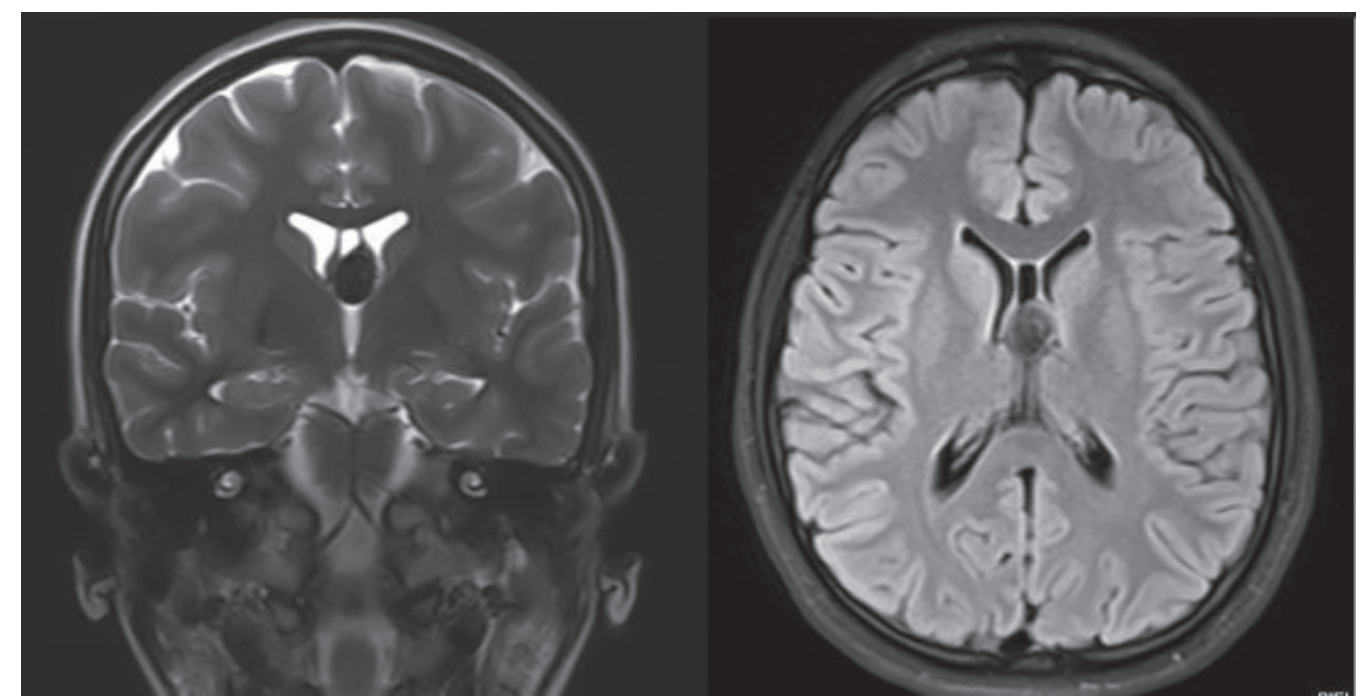

Fig. 2 Preoperative MRI coronal T2 and axial FLAIR showing colloid cyst of the CSP. CSP, cavumseptumpellucidum; FLAIR, fluid-attenuated inversion recovery; MRI, magnetic resonance imaging. 


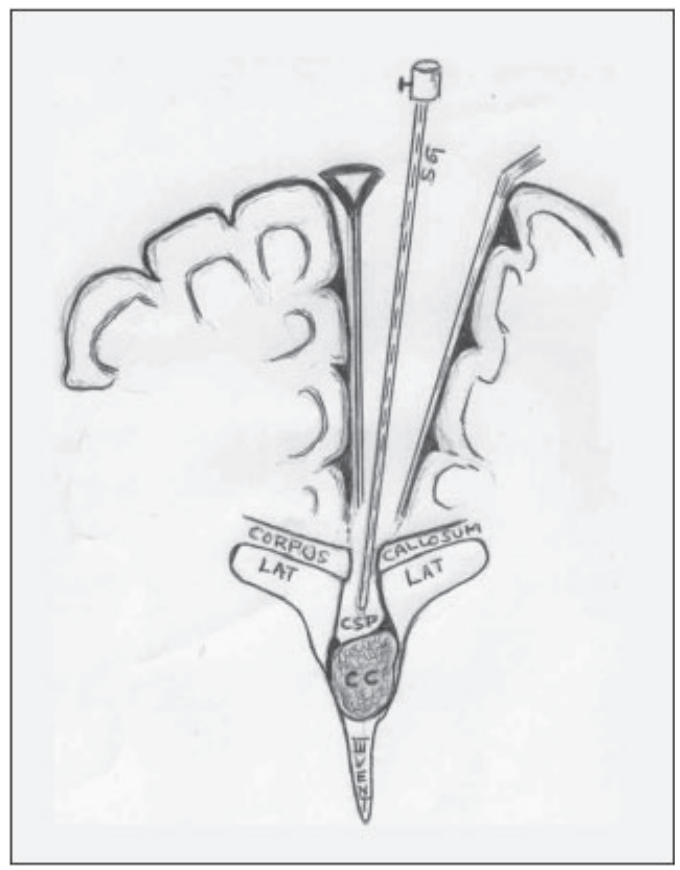

Fig. 3 Line diagram showing stereotactic approach to the lesion. CC, colloid cyst; CSP, cavum septum pellucidum; LAT, lateral ventricle; SG, stereotactic guidance; III VENT, third ventricle.

asymptomatic incidentally detected olloid cysts $(<1 \mathrm{~cm})$ are generally observed and followed up. The various theories of its origin include invagination or evagination of neuroepithelium from diencephalic roof during embryologic folding, as a product of early embryonal neuroectoderm, from choroid plexus, ependyma, paraphysis, or from primitive ectopic glial tissue in subarachnoid space. ${ }^{4}$

The operative approaches to colloid cyst utilize either microsurgical or endoscopic techniques, but either way, the

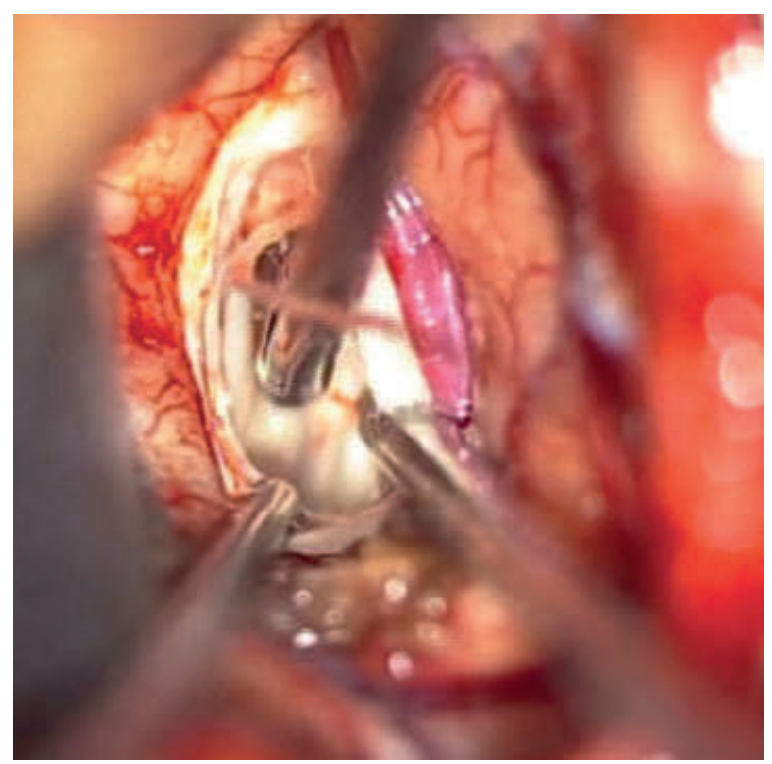

Fig. 4 Perioperative photo of microsurgical technique showing the stereotactic probe in the cavum septum pellucidum over the lesion. approach is directed to reach the foramen of Monro through the lateral ventricle, either transcortically or through transcallosum by following the choroid plexus. However, this approach might mislead to a negative exploration if the colloid cyst is encased within the leaves of the septum pellucidum.

Colloid cysts outside the third ventricle are extremely rare. Its location in the cavum septum pellucidum is very rarely reported. A colloid cyst in septum pellucidum location using Pantopaque ventriculogram was first reported by Ciric and Zivin in $1975 .{ }^{1}$ In their report of two cases, both the patients had hydrocephalus requiring shunt. Intraoperatively, transcortical transventricular approach was used. After entering the lateral ventricle, the foramen of Monro was not visualized due to the bulge of the septal leaf by the lesion. The septal leaf was incised subsequently and the lesion excised.

Koc (2007), ${ }^{3}$ in his case report, described a case of colloid cyst of the cavum septum pellucidum, which was approached endoscopically through right frontal horn. Intraoperatively, the septal leaf was opened and the colloid cyst excised.

Sadashiva et al $(2014)^{2}$ reported three cases of colloid cyst of the septum pellucidum, two of which were within the cavum septum pellucidum. In their first case, the lesion was approached through a transcallosal, transventricular dissection. On entering the lateral ventricle, the foramen of Monro was examined for the presence of the lesion but could not be found. Subsequently the septal leaf of cavum was opened to visualize the lesion that was then excised.

In their second case, the patient was initially operated elsewhere with transcallosal, transventricular approach to reach the foramen of Monro, but as the lesion was not found, the procedure was abandoned and subsequently referred to their center. Re-exploration of the patient showed a bulge on the septum which was opened to visualize the lesion and subsequently was excised.

In their third case, which was not associated with a cavum, on entering the lateral ventricle by transcallosal approach, the lesion was not seen near the foramen of Monro. They noticed a bulge in the septum was opened to visualize the lesion within the leaves of the septum pellucidum.

In all these case reports, the lesion was approached through the lateral ventricular cavity, where the lesion was not visualized near the foramen of Monro, and subsequently, on visualizing the bulge on the septum, which was incised before visualizing the lesion. In one of the cases, the surgery was abandoned due to nonvisualization of the lesion near the foramen of Monro.

In this case report, the use of stereotactic guidance, a routine practice in our unit for all deep-seated lesions, has actually helped us limit the extent of dissection of corpus callosum and enter directly the cavum without transgressing into the lateral ventricles. The surgical excision was easier in this approach when compared with the transventricular transforamen of Monro approach. Stereotactic guidance certainly has helped from being misled to reach the lesion. We claim that the search of colloid cyst near foramen of Monro is not necessary in this situation, and we also recommend the 


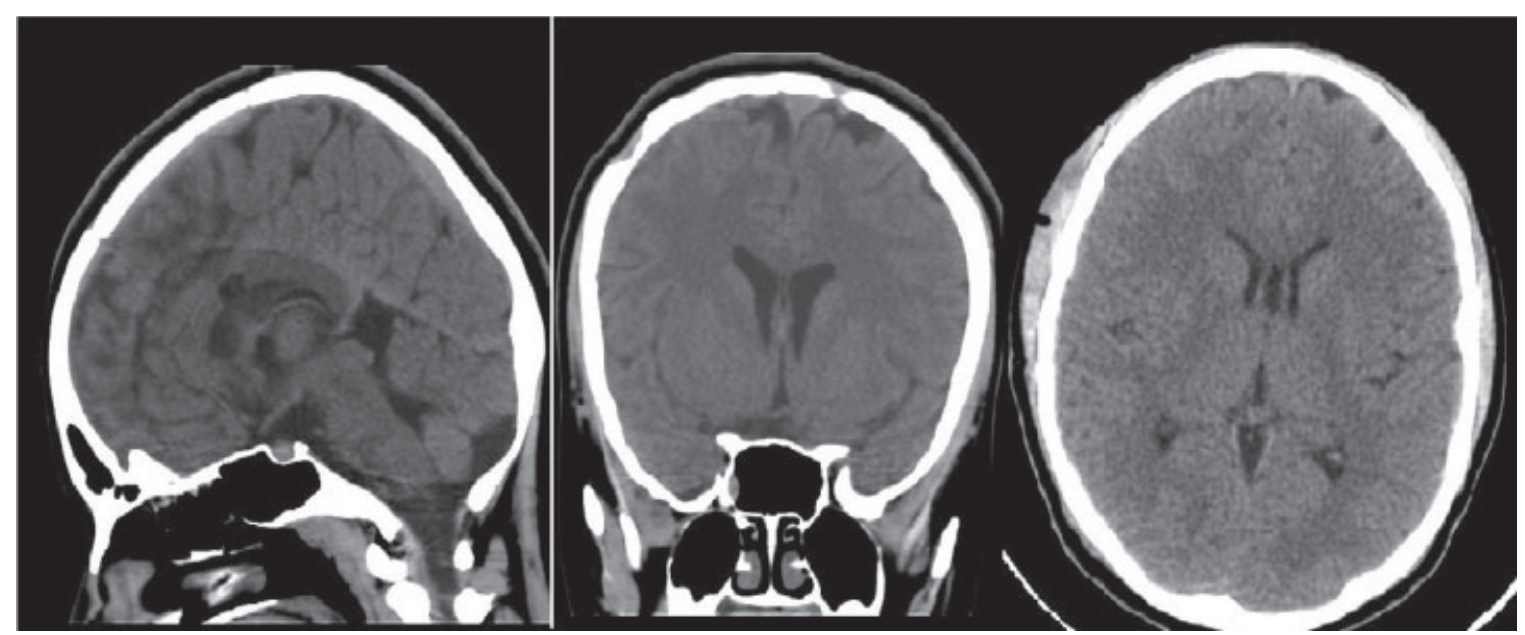

Fig. 5 Postoperative CT sagittal, coronal, and axial sections showing empty cavum septum pellucidum. CT, computed tomography.

routine use of stereotactic guidance to localize the lesions intraoperatively, which will obviously prevent failed surgeries or missed lesion since these lesions are usually small. This also minimizes the dissection of corpus callosum.

Intraoperative ultrasound-guided excision of colloid cyst ${ }^{5,6}$ has been reported in literature. The most important point to be noted here is its real-time value in identifying the lesion and operative guidance to the target. Adding echo Doppler will help in identifying the nearby vascular structures.

Frameless neuronavigation systems, ${ }^{7}$ which have been largely used of late, are beneficial in localizing the target, but they also have the same limitation as frame-based stereotactic guidance, especially in subcortical lesion. However, the brain shift after craniotomy in midline structures is not that significant when compared with the subcortical hemisp heric lesions.

\section{Conclusion}

Colloid cysts of the cavum septum pellucidum are scarcely reported in literature, and there is no defined approach to reach such location. The stereotactic-guided surgery will limit the area of exploration and provides a direct approach to the lesion in the cavum septum pellucidum, thus facilitating safe excision of the lesion and obviating negative explorations.

\section{References}

1 Ciric I, Zivin I. Neuroepithelial (colloid) cysts of the septum pellucidum. J Neurosurg 1975;43(1):69-73

2 Sadashiva N, Sastry S, Bhat D, Pandey P. Operative nuances of excision of colloid cysts in septum pellucidum: a report of three cases. Neurol India 2014;62(6):665-668

3 Koc K. Colloid cyst in cavum septum pellucidi: Rare location and endoscopic removal. J Neurol Sci Turish 2007;24:4

4 Efkan CM, Attar A, Ekinci C, Erdogan A. Neuroepithelial (colloid) cyst of the parietal convexity. Acta Neurochir (Wien) 2000;142(10):1167-1168

5 Rezvani L, Rubin JM, Dohrmann GJ. Colloid cysts with and without ventriculomegaly: role of intraoperative real-time ultrasound. Surg Neurol 1984;22(5):515-518

6 Filippini A, Prada F, Del Bene M, DiMeco F. Intraoperative cerebral ultrasound for third ventricle colloid cyst removal: case report. J Ultrasound 2014;19(3):211-215

7 Schroeder HWS, Wagner W, Tschiltschke W, Gaab MR. Frameless neuronavigation in intracranial endoscopic neurosurgery. J Neurosurg 2001;94(1):72-79 